\title{
New results on the distribution of thermal pressures in the diffuse ISM
}

\author{
Edward B. Jenkins ${ }^{1}$ and Todd M. Tripp ${ }^{2}$ \\ ${ }^{1}$ Princeton University Observatory, Princeton, NJ, USA \\ email: ebj@astro.princeton.edu \\ ${ }^{2}$ Department of Astronomy, University of Massachusetts, Amherst, MA, USA \\ email: tripp@fcrao1.astro.umass.edu
}

\begin{abstract}
The ground electronic state of neutral atomic carbon has three fine-structure levels. In the interstellar medium, the relative populations of the upper two levels are established by collisional excitations (and de-excitations) balanced against spontaneous radiative decay. Consequently, the fractions of C I in the upper two levels indicate acceptable combinations of local temperature and density, which in turn indicate the approximate thermal pressures of the medium. We can measure the values of these fractions and how they vary from one location to the next by observing the multiplets of $\mathrm{C}$ I seen in absorption in the ultraviolet spectra of hot stars.

We have identified 102 stars for which the HST MAST archive has E140H STIS spectra that are suitable for measuring the absorption features of C I at velocity resolutions of $3 \mathrm{~km} \mathrm{~s}^{-1}$ (or better). A special analysis method developed by Jenkins \& Tripp (2001) permits determinations of the amounts of $\mathrm{C} \mathrm{I}$ in each of the three levels as a function of radial velocity over a wide dynamic range in column density, since several multiplets of vastly different strengths can be considered simultaneously.

The C I data reveal that the much of the diffuse, cold, neutral medium has pressures that are distributed in an approximately log-normal fashion, spread over a range $1000<p / k<$ $10^{4} \mathrm{~cm}^{-3} \mathrm{~K}$ (FWHM), but with low level tails outside this range. The dispersion of pressures increases slightly for gases that have radial velocities outside the expected range for quiescent material along each line of sight. This link to the kinematics of the gas is consistent with the picture that pressure fluctuations are driven by the dynamics of a turbulent medium. If the gas is a single medium that is being driven by turbulent forces, its barytropic index (slope of $\log p$ vs. $\log n$ ) is more than 0.9 , which is inconsistent with the value 0.72 for material that is expected to be in thermal equilibrium. Slightly less than one part in a thousand of the gas is at pressures of order or greater than $\sim 10^{5} \mathrm{~cm}^{-3} \mathrm{~K}$ and seems to nearly always accompany the gas at normal pressures.
\end{abstract}

Keywords. ISM: atoms, ISM: general, techniques: spectroscopic, turbulence

\section{Introduction}

One of the challenges in observing the interstellar medium (ISM) is to sense local physical conditions in space, an exercise which goes beyond the much simpler task of determining the amounts of various constituents integrated along lines of sight. One excellent indicator of local conditions in the diffuse, neutral gas is the neutral carbon atom (C I), which has a ground electronic state that is split into three fine-structure levels, two of which have excitation energies $E / k=23.6$ and $62.4 \mathrm{~K}$ above the lowest level. These upper two levels, which we designate as $\mathrm{C} \mathrm{I}^{*}$ and $\mathrm{C} \mathrm{I}^{* *}$, are populated by collisions with other gas components. Since these excitations compete with spontaneous radiative decay, the equilibrium concentrations of the excited levels indicate local kinetic temperatures and densities. One can measure these relative populations by observing an 
array of C I multiplets in the ultraviolet region, which show up as absorption features in the spectra of bright background sources. One important feature of these multiplets is that they have line strengths that span 3 orders of magnitude, which offers a large dynamic range in the measurements of column densities.

There have been numerous investigations of the C I excitation in the general ISM, in specific sites of interest (e.g., shocked clouds inside supernova remnants), and for very distant gas systems that are seen in absorption against quasars. The echelle spectrograph of the STIS instrument on the Hubble Space Telescope, when used in its highest resolution mode, offers exquisite recordings of the C I multiplets that appear in the spectra of earlytype stars. In an earlier survey, we made use of this instrument to observe C I absorption toward 21 stars with a velocity resolution of $1.5 \mathrm{~km} \mathrm{~s}^{-1}$ (Jenkins \& Tripp 2001). We have now built upon this investigation by analyzing the spectra of 81 additional stars obtained from the HST MAST archive, and we report the initial conclusions from this new effort here. The resolution of nearly all of these additional stellar spectra, about $3 \mathrm{~km} \mathrm{~s}^{-1}$, is lower than our original survey because the default slit width of 0.09 arc-sec for the E140H mode was used instead of the extra narrow slit with a width of 0.03 arc-sec.

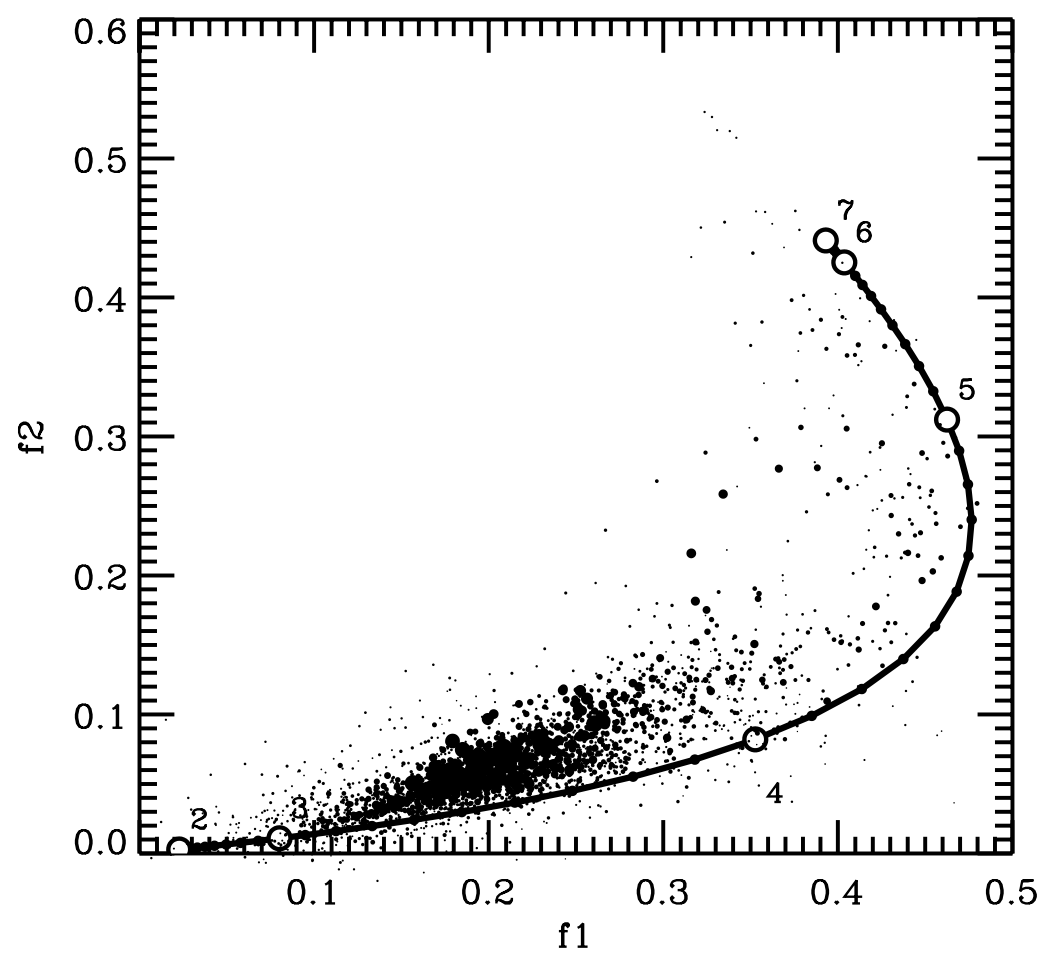

Figure 1. Values of $f 1$ and $f 2$ for all measurements of $\mathrm{C} I$ in the survey of 102 stars. The area of each dot indicates the total column density of C I (in all three of the fine-structure levels). The curve indicates the loci of $f 1$ and $f 2$ values for homogeneous regions at a temperature $T=100 \mathrm{~K}$ for various values of thermal pressure $p / k$ (integer values of $\log [p / k]$ are indicated by open circles with numbers showing these values; bead-like dots on the curve show increments of 0.1 dex.). Other values of $T$ yield curves similar to this one - see Fig. 6 of Jenkins \& Tripp (2001). The fact that most of the points fall inside of rather than on the arc indicates that we usually are sensing gas with a small admixture of high pressure material - see Jenkins \& Tripp (2001, 2007) for an explanation of how to interpret this effect. 

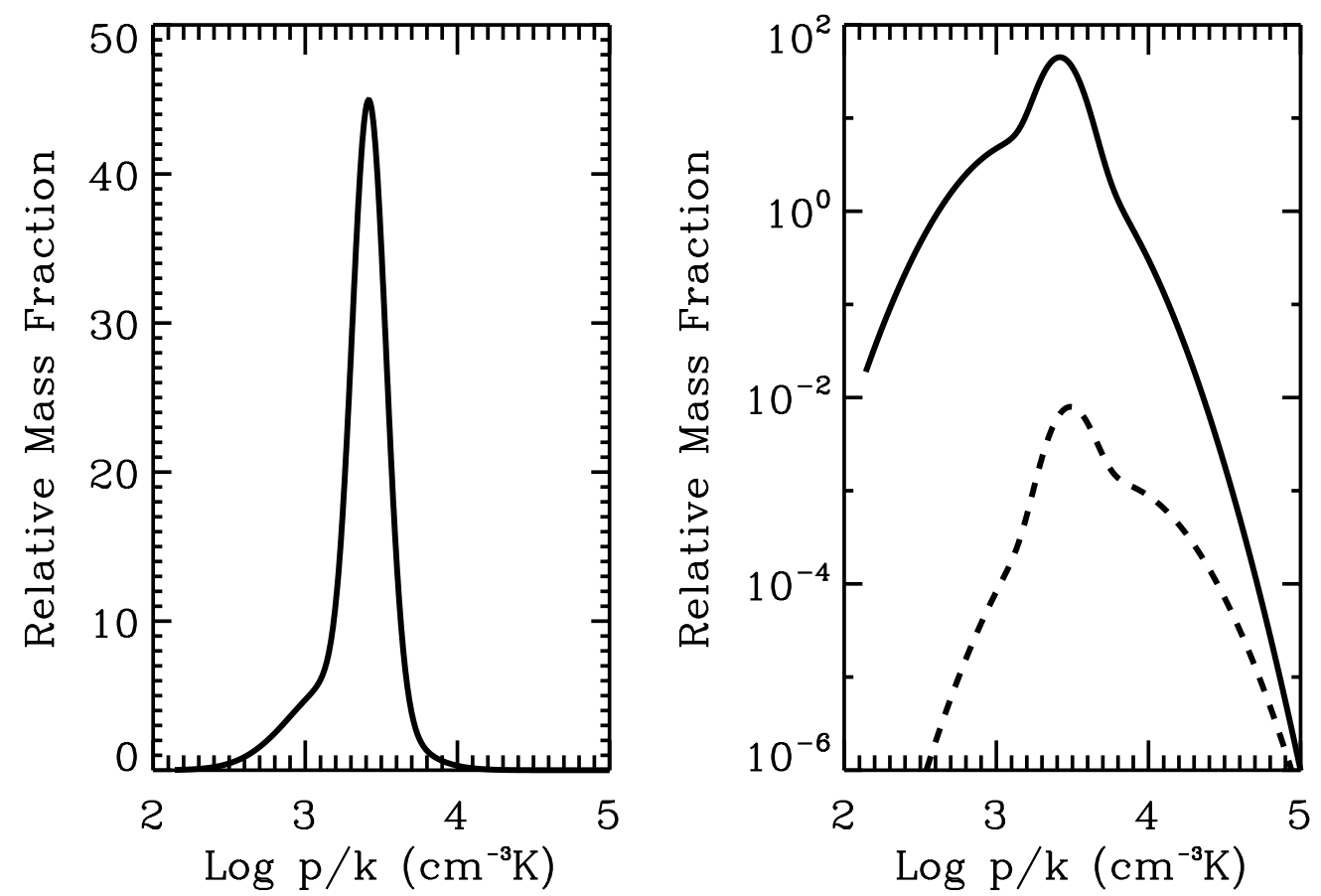

Figure 2. Linear and logarithmic plots of the distribution by mass of thermal pressures of hydrogen gas in the diffuse, neutral medium between us and the target stars in the survey. The dashed curve in the right-hand (logarithmic) display indicates the approximate proportion of gas at $p / k \sim 10^{5} \mathrm{~cm}^{-3} \mathrm{~K}$ that accompanies the gas at the indicated value of $p / k$ in the plot. This high pressure component is needed to explain the displacement of the points above the lower portion of the curve shown in Fig. 1.

\section{Analysis and results}

The normal range of velocity spreads in typical ISM sight lines cause different lines in the C I multiplets to overlap each other. To overcome the confusion caused by these blends, we developed (and described in our 2001 paper) an analysis method that derived solutions for the velocity profiles of each of the three levels that would recreate the patterns seen in the different multiplets. We then calculated for all velocities where measurable absorptions were present two excitation parameters $f 1=N\left(\mathrm{C} \mathrm{I}^{*}\right) / N\left(\mathrm{C} \mathrm{I}_{\text {total }}\right)$ and $f 2=N\left(\mathrm{C} \mathrm{I}^{* *}\right) / N\left(\mathrm{C} \mathrm{I}_{\text {total }}\right)$, where $N\left(\mathrm{C} \mathrm{I}_{\text {total }}\right)=N\left(\mathrm{C} \mathrm{I}_{\text {unexc. }}\right)+N\left(\mathrm{C} \mathrm{I}^{*}\right)+N\left(\mathrm{C} \mathrm{I}^{* *}\right)$.

In Figure 1 we show the determinations of $f 1$ and $f 2$ for all of the $0.5 \mathrm{~km} \mathrm{~s}^{-1}$ wide velocity bins toward all of the targets in the survey. Most of the points fall within the range $3.0<\log (p / k)<4.0$ of the nearby arc that depicts the expected values of $f 1$ and $f 2$ for $T=100 \mathrm{~K}$. In Figure 2 we show the distribution of pressures that is consistent with the locations of the points shown in Fig. 1. In deriving this distribution, we had to calculate shifts in the ionization equilibrium between the neutral and ionized forms of $\mathrm{C}$ and correct for this effect to obtain the H I-weighted distribution.

We interpret the displacement of the points above the lower portion of the arc in Fig. 2 to arise from either an ever present admixture of a very small amount of gas at very high pressures $\left(p / k \gtrsim 10^{5} \mathrm{~cm}^{-3} \mathrm{~K}\right)$ or excitations by positively charged particles that can more strongly excite the C I** [see Fig. 1 of Silva \& Viegas (2002)]. In weakly ionized regions, 
excitation from shocks or Alfvén waves could create differences in the velocities of ions and neutrals, which in turn could excite the carbon atoms.

As emphasized in our earlier work (Jenkins \& Tripp 2001), the points in the higher parts of the $f 1-f 2$ diagram represent gas at high pressures $\left(p / k>10^{4} \mathrm{~cm}^{-3} \mathrm{~K}\right)$, but at temperatures not much below about $80 \mathrm{~K}$. This indicates that the material does not cool appreciably when it is compressed, which would happen if the process were slow enough to allow a thermal equilibrium to be maintained $\left[d \log p / d \log n=\gamma_{\mathrm{eff}}=0.72\right.$ (Wolfire, et al. 1995)]. For this reason, we favor the interpretation that the gas is compressed so quickly that the changes in temperature are closer to adiabatic $(\gamma=5 / 3)$. It should not be difficult for turbulent motions to achieve such compressions if the gas regions have sizes less than about $0.01 \mathrm{pc}$.

When we examine the kinematics of the gas, we find that material at velocities outside the interval expected for differential galactic rotation along the sight line has a greater tendency to show high pressures. The evidence for this is illustrated in Jenkins \& Tripp (2007), and it is consistent with the proposition that shocks and colliding flows of gas are the probably cause for episodic enhancements of the thermal pressure.

\section{Acknowledgements}

This research was supported by grant HST-AR-09534.01 from the Space Telescope Science Institute, operated by the Association of Universities for Research in Astronomy, Inc., under NASA contract NAS5-26555.

\section{References}

Jenkins, E. B. \& Tripp, T. M. 2001, ApJS 137, 297

Jenkins, E. B. \& Tripp, T. M. 2007, in: M. Goss \& M. Haverkorn (eds.), Small Ionized and Neutral Structures in the Diffuse Interstellar Medium (ASP-CS), in press

Silva, A. I. \& Viegas, S. M. 2002, MNRAS 329, 135

Wolfire, M. G., Hollenbach, D., McKee, C. F., Tielens, A. G. G. M. \& Bakes, E. L. O. 1995, ApJ 443, 152

\section{Discussion}

M. Dopita: The distributions you showed have both a broad and a narrow log-normal curve. Are the broad components really separate phases of the ISM?

E. Jenkins: Offhand, I see no easy way to determine if this is the case. Generally, the C I ionization equilibrium strongly enhances our sensitivity to the cold, neutral medium, at the expense of the warm neutral, or partially ionized medium.

B. Elmegreen: Do you see a correspondence between enhancements in the ratio of Ca II vs. Na I and elevated thermal pressures, as indicated by C I?

E. Jenkins: This is an interesting idea. We have not investigated this issue, but perhaps we should do so. 